\title{
Electric source localization adds evidence for task-specific CNVs
}

\author{
Ina M. Tarkka ${ }^{\mathrm{a}, *}$ and Luis F.H. Basile ${ }^{\mathrm{b}}$ \\ ${ }^{a}$ Brain Research and Rehabilitation Center Neuron, \\ Kuopio, Finland \\ ${ }^{\mathrm{b}}$ Department of Psychiatry, University of Sao Paulo \\ Medical School, Sao Paulo, Brazil
}

Received 1 March 1997

Revised 19 February 1998

This study was an attempt to replicate recent magnetoencephalographic (MEG) findings on human task-specific CNV sources (Basile et al., Electroencephalography and Clinical Neurophysiology 90, 1994, 157-165) by means of a spatio-temporal electric source localization method (Scherg and von Cramon, Electroencephalography and Clinical Neurophysiology 62, 1985, 3244; Scherg and von Cramon, Electroencephalography and Clinical Neurophysiology 65, 1986, 344-360; Scherg and Berg, Brain Electric Source Analysis Handbook, Version 2). The previous MEG results showed CNV sources in the prefrontal cortex of the two hemispheres for two tasks used, namely visual pattern recognition and visual spatial recognition tasks. In the right hemisphere, the sources were more anterior and inferior for the spatial recognition task than for the pattern recognition task. In the present study we obtained CNVs in five subjects during two tasks identical to the MEG study. The elicited electric potentials were modeled with four spatio-temporal dipoles for each task, three of which accounted for the visual evoked response and one that accounted for the CNV. For all subjects the dipole explaining the $\mathrm{CNV}$ was always localized in the frontal region of the head, however, the dipole obtained during the visual spatial recognition task was more anterior than the one obtained during the pattern recognition task. Thus, task-specific CNV sources were again observed, although the stable model consisted of only one dipole located close to the midline instead of one dipole in each hemisphere. This was a major difference in the CNV sources between the previous MEG and the present electric source analysis results. We discuss the possible basis for the difference between the two methods used to study slow brain activity that is believed to originate from extended cortical patches.

Keywords: Prefrontal cortex, CNV, electric source analysis, magnetoencephalography, visual recognition tasks

${ }^{*}$ Corresponding author: Ina M. Tarkka, Ph.D., Brain Research and Rehabilitation Center Neuron, Kortejoki, FIN-71130 Kuopio, Finland. Tel.: +358 17460281; Fax: +358 17460200 .

Behavioural Neurology 11 (1998) 21-28

ISSN 0953-4180 / \$8.00 (C) 1998, IOS Press. All rights reserved

\section{Introduction}

The contingent negative variations (CNVs) are negative shifts of electric potentials recorded on the centro-frontal scalp that are elicited when a subject expects events that must be evaluated for possible use in a given context $[19,20,8]$. A recent magnetoencephalographic study [1] has shown that the CNVs are generated in the prefrontal cortex, and seem to originate from different areas within the prefrontal cortex depending on the type of task performed by subjects. Dipole-like sources of the magnetic equivalents of CNVs obtained during a visual spatial recognition task were found in more anterior and inferior regions of the right prefrontal cortex when compared with dipoles found during a visual pattern recognition task [1]. Those results agree with a recent study on other primates [22] that demonstrated a concentration of task-specific neurons in two homologous areas of the prefrontal cortex, depending on whether the animals performed a visual spatial or a pattern recognition task.

The purpose of the present study was to test whether currently available source analysis methods for electrical data could be used to model the sources of the CNVs, and distinguish the sources corresponding to $\mathrm{CNV}$ s elicited during the performance of different tasks. With the use of 30 scalp recordings, averaged reference and a spatio-temporal modeling approach with multiple dipoles $[14,15,16]$, we expected to obtain analogous results to our MEG studies, although some differences were anticipated; the spacial summation of the recorded electric potentials was expected to be higher than of the recorded magnetic fields owing to the differences between the sensitivities of the EEG and MEG methods to electromagnetic changes in the brain.

The tasks used in the present study were identical to those used in our first study on the magnetic equivalents of CNVs [1]. The tasks consisted of visual spatial and visual pattern recognition, both to be performed with the use of a common set of visual stimuli. 


\section{Place Recognition}

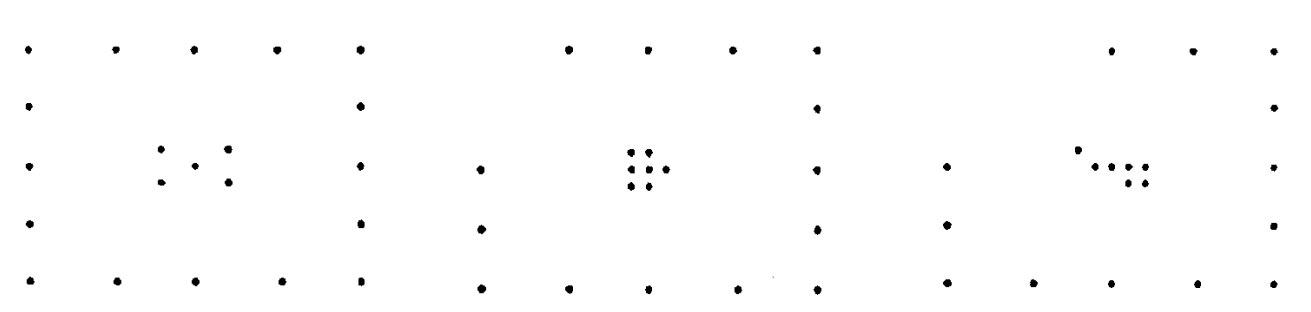

\section{Pattern Recognition}

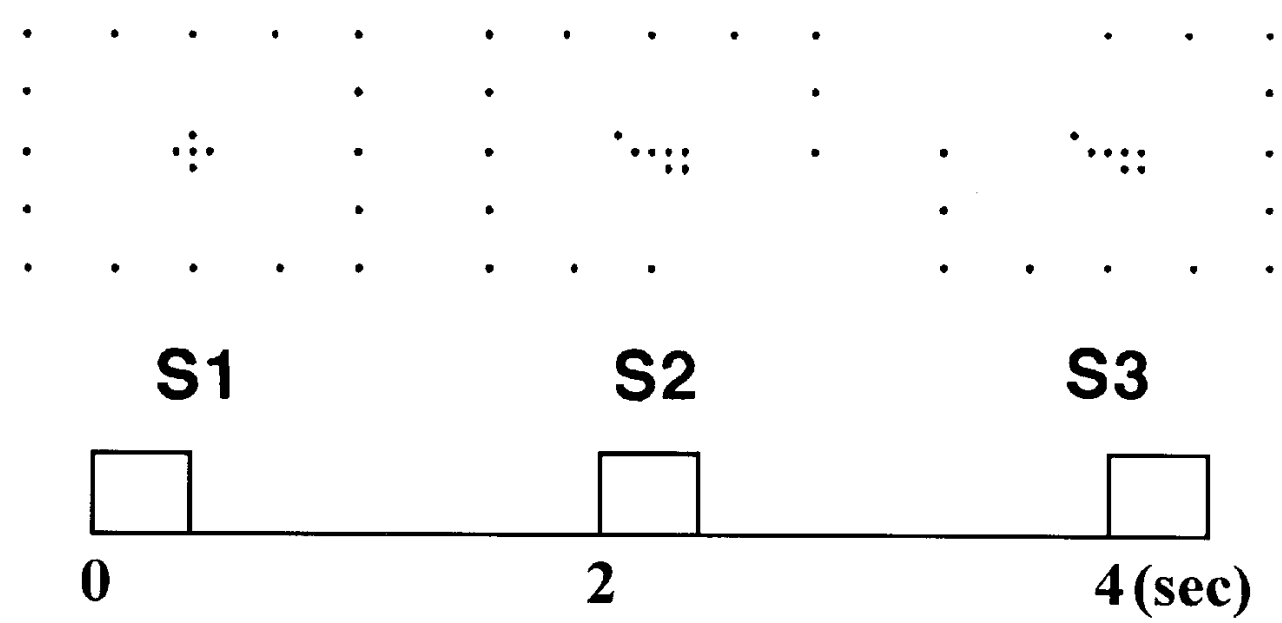

Fig. 1. Examples of stimuli used in the two types of trials. The warning stimulus (S1) was specific to each task. For more details see the text. Both trials shown above required 'same' as the correct response.

\section{Methods}

\subsection{Subjects}

Five healthy subjects, 3 males and 2 females, ranging in age from 28 to 38 years, volunteered to participate in the study. All subjects had normal vision and no history of neurological disorders. They were not taking any medications and reported no substance abuse. The protocol was approved by the Internal Review Board for Human Research and each subject signed a consent form prior to the study.

\subsection{Tasks}

The subject was seated comfortably in a dark room facing a board ( $2 \mathrm{~m}$ distance) with light dots controlled by a computer. The visual stimuli were built with an array of light dots subtending approximately 25 degrees of the visual field (see Fig. 1). Subjects were instructed to fixate their gaze on a light dot that was kept on in the center of the board throughout each test run.
On a given trial, one of the two possible warning stimuli (S1) indicated to the subject which of the two tasks (spatial or pattern recognition) had to be performed on the two stimuli that were subsequently presented during each trial (S2 and S3). Those S2 and S3 stimuli had to be compared. The arrangement of six out of fourteen possible light dots defined the central visual field portion of the stimuli, composing the visual patterns (3 degrees of eccentricity), to be attended to during the pattern recognition task. During that task, the pattern presented in one stimulus of each trial (S2) had to be compared with the pattern presented in the subsequent stimulus of that trial (S3). When the warning stimulus indicated that the subject should engage in the spatial task, i.e., a place recognition task, then a rectangular array of lights in the peripheral visual field had to be attended to and compared in S2 and S3. On each stimulus one of the corners in the peripheral array of lights contained light dots that were turned off during stimulus presentation. Those corners with missing light points were the places to be recognized 
and compared, between the S2 and S3 stimuli during the place recognition task. Thus, at S1 presentation the subjects were to prepare to attend to the peripheral field or to the central field shown in the subsequently presented S2 and S3 stimuli. Then they had to decide whether the corner containing missing lights (or central field pattern) shown in the S2 stimulus matched the corner (or pattern) shown in the S3 stimulus. They were required to respond verbally saying 'same' or 'different' to indicate a match or mismatch between the relevant aspects of S2 and S3, soon after the presentation of the $\mathrm{S} 3$ stimulus. The subjects' responses were recorded. Fifty percent of the trials comprised the place recognition and fifty percent the pattern recognition task. The order of trials was randomized within each session. The exposure duration of each stimulus was $300 \mathrm{~ms}$ followed by a masking stimulus with all light dots turned on for $50 \mathrm{~ms}$ (see Fig. 1). Within a trial, the inter-stimulus interval was $2000 \mathrm{~ms}$ and the intertrial interval was $5 \mathrm{~s}$.

\subsection{Recording}

Event-related potentials (ERP) were recorded with 30 conventional EEG electrodes $(\mathrm{Ag} / \mathrm{AgCl}$ disk electrodes, diameter $10 \mathrm{~mm}$ ) placed on the scalp according to the modified International 10-20 System where more electrodes were placed over the forehead than over parietal scalp areas (see Fig. 2). The reference during recording was the linked earlobe electrode and the ground electrode was attached to the right wrist. EOG was monitored throughout. The electrode impedance was kept below $3 \mathrm{k} \Omega$ throughout the entire recording.

The amplifier bandpass extended from 0.07 to $40 \mathrm{~Hz}$ with a recording window of $3000 \mathrm{~ms}$, of which $1000 \mathrm{~ms}$ was the baseline and a $2000 \mathrm{~ms}$ window was analyzed. Sweeps from the pattern recognition task and from the place recognition task were averaged into separate buffers collecting about 60 artefact-free sweeps for each average (Dantec Concerto) using an automatic artefact rejection program. The impedance control, eye movement control and the similar number of sweeps for both tasks ascertain data reliability as far as it is possible with electrical scalp potentials. The data were transferred to a personal computer for Brain Electrical Source Analysis (BESA). Specific instructions were given to subjects to avoid blinking and facial movements during the trials.
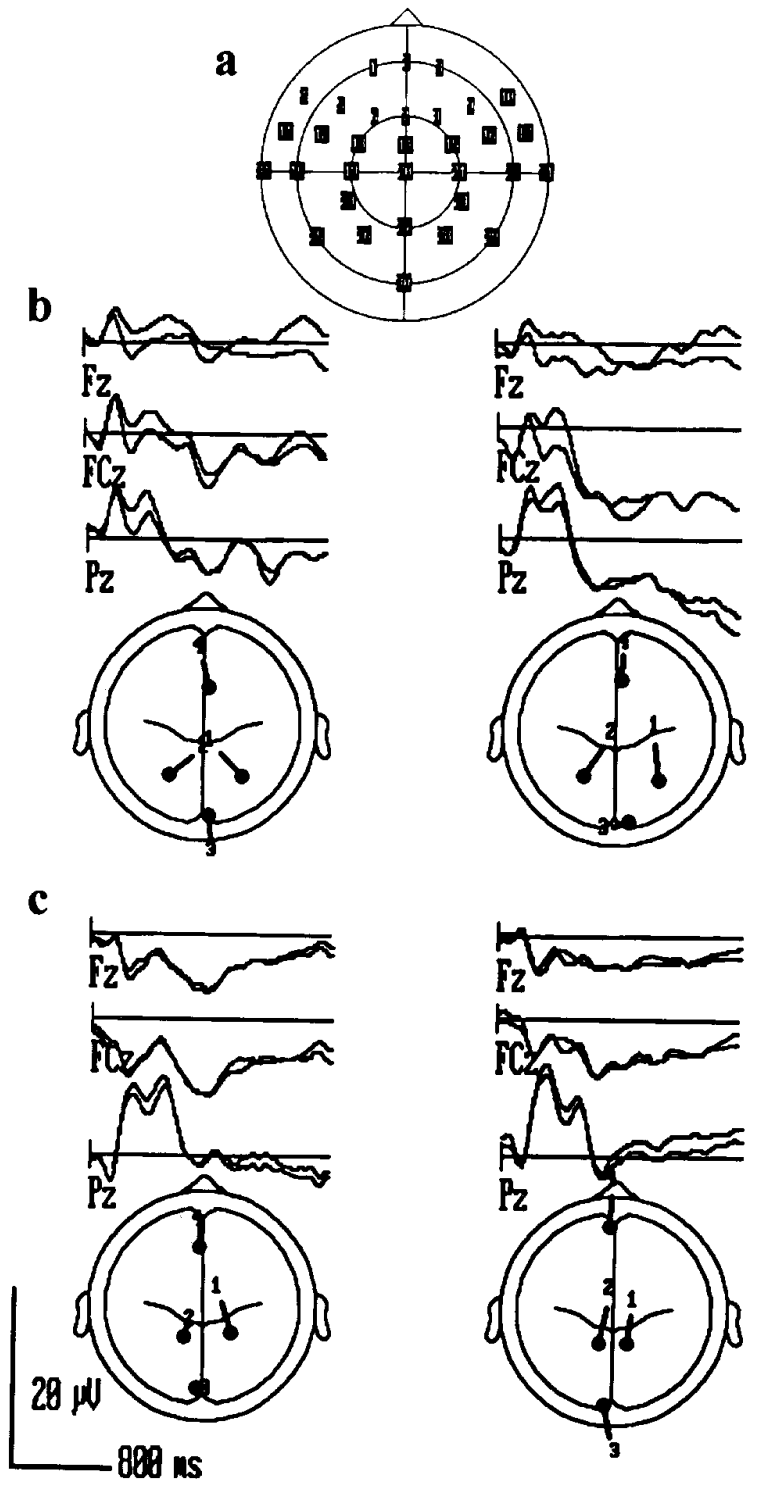

Fig. 2. A schema showing the electrode placement on the scalp in a. CNVs associated with the visual pattern and place recognition tasks in $\mathbf{b}$ and $\mathbf{c}$. The examples of recorded waveforms and the waveforms created by the models for the same locations are superimposed and in addition, the respective 4-dipole models are viewed from the top of the head in two individual subjects. Visual pattern recognition $\mathrm{CNV}$ and place recognition CNV for one subject in $\mathbf{b}$ and for another subject pattern recognition $\mathrm{CNV}$ and place recognition $\mathrm{CNV}$ in $\mathbf{c}$. Note that the dipole 4 in the frontal region appears more anterior in the models explaining the place recognition tasks (models on the right).

\subsection{Multiple source localization method}

The BESA technique developed by Scherg $[14,16]$ allows the researcher to generate an equivalent spatiotemporal dipole model of multiple source generators 
from the recording of the scalp surface ERP. We used a theoretical spherical head model of four shells [2]. The radius of the head of each individual subject was measured using a radio-frequency transmitting device (BTi, Inc.). The distance between the T3 and T4 electrode locations was measured and divided by two. This value became the radius and the individual values were utilized in modeling each subject's data. The head radii varied from $7.0 \mathrm{~cm}$ to $7.9 \mathrm{~cm}$ among our subjects.

The BESA program allows progressive iterative fitting of the dipole parameters. As it is well known that no unique solution exists for the inverse problem, the strategy for model development is important. Before any attempts were made to develop a model for the CNVs, current source density maps (i.e., a Laplacian operator was used to map the voltage distribution of each time point) were generated. Mapping was done for the data of each individual, for both tasks and for the duration of $980 \mathrm{~ms}$ for each sampled time point starting from $1020 \mathrm{~ms}$ after stimulus onset. Current source density maps allow a rough estimation of source locations at instantaneous time points. The mapping results gave us sufficient confidence to develop spatiotemporal multiple dipole models. The approach for the model development was the following: because of the slow ERP activity, which may not return to the baseline, we included the visual evoked potential elicited by the stimulus and the following P3-like positive component into the window to be modeled. Thus, a full $2000 \mathrm{~ms}$ window with a relatively low sampling rate was modeled. Even though early activity was included in the analyzed sweep, our aim was to model primarily the source(s) for the CNV and not to concentrate on the source(s) of the preceding activity. Thus, after achieving stable models for each subject we analyzed in detail only the parameters describing the dipoles active during the CNV period.

In order to start the modeling procedure, grand average waveforms from the data of all subjects with $2000 \mathrm{~ms}$ window were created for the two types of CNVs. These grand average waveforms were modeled using a generic head model with a radius of $85 \mathrm{~mm}$ in an attempt to find the minimum number of dipoles sufficient to explain the waveforms. The modeling ideology of BESA requires finding the least number of dipoles to explain the data. It is important to avoid explaining signal noise. Grand averaging reduces noise in the signal and thus it is easier to find the minimum number of dipoles than in the case of data from an individual subject. Stable models were found through test- ing with an additional dipole which, when the model is stable, does not attract potential.

The parameters of each dipole of the grand average models, location and orientation, were used as starting values for modeling the data of individual subjects with individual head radius values. Then, the two models for the two CNVs of each subject were optimized and fine-tuned using separation constraints and iterative fitting of individual dipoles. Finally, the locations of those dipoles predominantly active during the CNVs were compared within each subject between the place and pattern recognition tasks.

\section{Results}

Current source density maps were created for each individual and for both tasks at 5 instantaneous time points starting at $1020 \mathrm{~ms}$ after the $\mathrm{S} 1$ stimulus presentation. The maps of the place and pattern recognition task CNVs differed in each subject. The topographic patterns of the maps clearly suggested more anterior source(s) for the place recognition task than for the pattern recognition task in three subjects.

The grand average data was used to start developing the multiple dipole models. In the process, models having 2-6 dipoles were tested. However, 4 dipoles, 1 in the occipital lobe, 2 in each temporal region and 1 in the frontal lobe, proved to be the most stable model. Several other models were considered poor in explaining the data. Specifically, introducing 2 dipoles in the occipital lobe produced a fusion of those dipoles; the attempt to use only 1 dipole for deep temporal lobe activity produced high residual variances; and attempts to model frontal lobe activity with 2 or 3 dipoles consistently produced 1 dipole collecting almost all the activity and the 1 or 2 others attracting only negligible dipole moments. The 4-dipole model was confirmed by adding a fifth freely moving dipole to a stable model with lower than $6 \%$ residual variance.

Since this additional dipole did not attract significant dipole moment in either task, we concluded that 4 was a sufficient number of dipoles. Four-dipole models from both grand average datasets were given as starting values for the model development for individual data. The dipoles were fitted for both tasks in each subject using individual head radius values and a 4-shell spherical head model. Models for both tasks consisted of 2 temporal lobe dipoles, 1 occipital dipole and 1 frontal lobe dipole (see Fig. 2). The occipital lobe dipole was active starting at about $60 \mathrm{~ms}$ for a pe- 
a

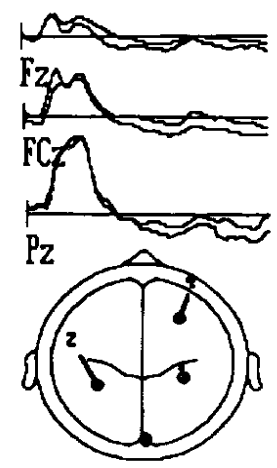

b

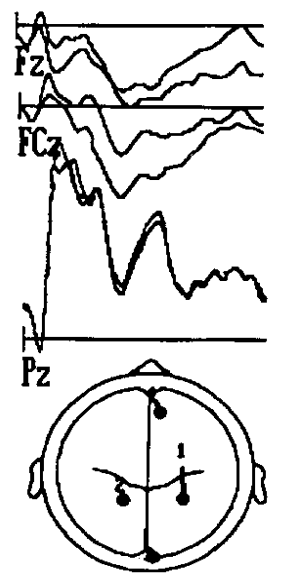

c

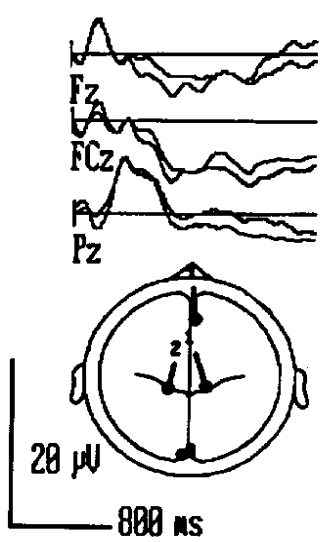

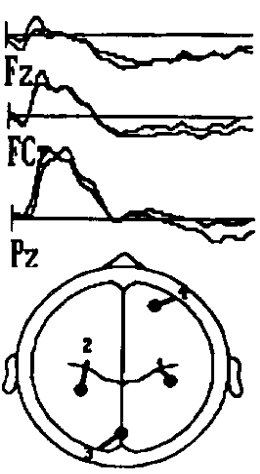
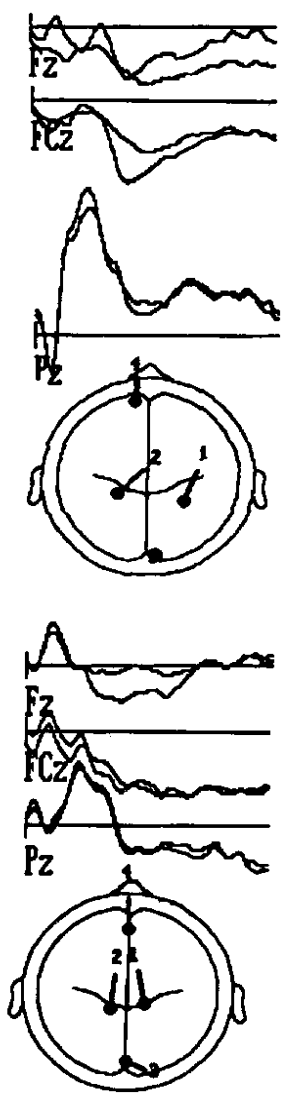

Fig. 3. CNVs associated with the visual pattern and place recognition tasks in three other subjects. The example waveforms and corresponding model waveforms are superimposed and the 4-dipole models are shown in a view from the top. On the left are the pattern recognition $\mathrm{CNV}$ s and models and on the right are the place recognition CNVs and models; $\mathbf{a}, \mathbf{b}$ and $\mathbf{c}$ indicate individual subjects.

riod of 200 to $300 \mathrm{~ms}$, depending on the subject. The temporal lobe dipoles were bilaterally active starting around $150 \mathrm{~ms}$ for 500 or $600 \mathrm{~ms}$. The frontal lobe

dipole, obtained in the right side of the head in all but one occasion, began to show activity around $700 \mathrm{~ms}$ and continued to be active until $1500 \mathrm{~ms}$, decreasing gradually thereafter.

The occipital dipole location, orientation and the period of activation suggest that it accounts for activity in the primary visual area evoked by the stimulus presentation. Visual evoked potential, its waveform shape and the explaining dipole location, is well known from previous work. Also, the temporal separation of the visual evoked potential and the CNV allows us to concentrate only on CNV. The temporal lobe dipoles, with slightly different locations across the subjects (see Figs 2 and 3), account for the P3-like activity following the warning stimulus presentation. The approximate locations of these dipoles generally agree with more detailed noninvasive observations of the possible electrical source locations of P3s [10, 11, 12, 17, 18]. The parameters of the occipital and temporal dipoles were not further analyzed since our goal was to model the CNVs. Using individual head radii for the sphere and the 4-shell model improved the modeling, producing more stable models as compared with previous modeling attempts using a fixed head radius or a 3-shell head model.

Fig. 2 illustrates the waveforms and the developed models for the pattern and place recognition tasks in two subjects. The recorded data waveforms and waveforms produced by the model are superimposed. The difference between the data and the model waveforms is described by the residual variance (RV). RV was calculated for the complete analyzed window of $2000 \mathrm{~ms}$. $\mathrm{RV}$ values ranged from $4-6 \%$. The dipole models are seen from the top of the head in order to illustrate the anterior-posterior difference of the dipole 4, i.e., the prefrontal dipole active during both types of CNVs. Fig. 3 illustrates the recorded waveforms, the superimposed model waveforms and the achieved models in a top view for the other three subjects. Some electrode locations show more differences than others, however the RV describes the goodness of fit throughout the electrode montage. Some locations have more noise than others, such as low temporal electrodes.

The fourth dipole explaining the CNVs was located more anterior for the place recognition task CNV than for the pattern recognition task CNV. The more anterior location was the case for all subjects although the magnitude of the difference varied from subject to subject. The differences in anterior direction were tested between tasks with a sign-test and were significant. Slight changes in the dipole locations between 
Table 1

Comparison of the locations of dipole 4 , active during the CNV period, for the pattern and the place recognition tasks within each subject $^{a}$

\begin{tabular}{|c|c|c|c|c|c|}
\hline & & $X$ & $\mathrm{Y}$ & $\mathrm{Z}$ & $\begin{array}{l}\text { Spatial } \\
\text { distance }\end{array}$ \\
\hline \multirow[t]{3}{*}{$\mathrm{NC}$} & pattern & 3.9 & 27.9 & 12.0 & \\
\hline & place & 3.4 & 37.6 & 33.6 & \\
\hline & $\Delta$ & 0.5 & 9.7 & 21.6 & 24 \\
\hline \multirow[t]{3}{*}{ DS } & pattern & -2.3 & 40.5 & 27.6 & \\
\hline & place & -3.9 & 57.4 & 28.5 & \\
\hline & $\Delta$ & 1.6 & 16.9 & 0.9 & 17 \\
\hline \multirow[t]{3}{*}{ LB } & pattern & 26.3 & 33.8 & 11.4 & \\
\hline & place & 22.4 & 47.0 & 8.2 & \\
\hline & $\Delta$ & 3.9 & 13.2 & 3.2 & 14 \\
\hline \multirow[t]{3}{*}{$\mathrm{JL}$} & pattern & 27.7 & 43.5 & 0.4 & \\
\hline & place & -11.0 & 60.6 & 20.1 & \\
\hline & $\Delta$ & 38.7 & 17.1 & 19.7 & 47 \\
\hline \multirow[t]{4}{*}{$\mathrm{IM}$} & pattern & 2.8 & 40.8 & 7.6 & \\
\hline & place & -0.7 & 44.8 & 20.2 & \\
\hline & $\Delta$ & 3.5 & 4.0 & 12.6 & 14 \\
\hline & Mean $\pm \mathrm{SD}$ & & & & $23 \pm 14$ \\
\hline
\end{tabular}

${ }^{a} \mathrm{X}$-axis; positive values towards the right preauricular point, Yaxis; positive values towards the nasion and Z-axis; directs towards $\mathrm{CZ}$. Values in $\mathrm{mm}, \Delta$ indicates the difference between pattern and place recognition tasks.

the tasks were also observed in the other two directions but not in a very systematic manner. The differences in dipole locations for each subject are detailed in Table 1. The CNV dipoles were located close to the midline, on the right side of the head. The table shows interindividual differences both in dipole locations and in the spatial distance between the dipoles of the two conditions. The main reason for interindividual differences is different head sizes which shows because the head models were individualized with measured head radii. Also, the brain anatomy certainly differs between these subjects. Thus, the varying numbers reflect the differences in brains and active locii and as numerical values are not very revealing. The mean spatial distance of the CNV dipoles between the two tasks was $23 \pm 14 \mathrm{~mm}$.

\section{Discussion}

The present results demonstrate that engagement of subjects in the two visual tasks was associated with the generation of the two task-specific CNVs. For all five subjects, the sources of the two types of CNVs, each corresponding to one task, were modeled by one equivalent current dipole centered at a frontal region of the head. The visual spatial recognition task corresponded to activity that had its sources explained by a dipole centered at a more anterior position than the dipole that was obtained in the model for the CNV of the pattern recognition task. This was true for all subjects and is in general agreement with our previous magnetoencephalographic findings [1], in which more anterior sources were obtained for the spatial task. The time period in the original data where the best solutions for the source localization algorithms were found occurred around 1 second after the stimulus presentation, common to both EEG and MEG studies. However, one important difference between the results from the two methods was observed. That is, with MEG two dipole-like sources were obtained during performance of each task, one dipole in each hemisphere, corresponding to clearly separated pairs of magnetic field extrema on the scalp. In contrast, in the present EEG study we observed only one dipole accounting for the electric potentials obtained for each task, localized typically in the right hemisphere but close to the midline of the head.

The difference between MEG and EEG source localization results was not surprising, since it is well known that MEG and EEG detect different aspects of a given pattern of electrical activity in the brain. Briefly, MEG is almost exclusively sensitive to tangential primary (cortical pyramidal) currents and the magnetic fields are practically unaltered when crossing the different tissues of the head $[21,4,7]$. EEG, in contrast, detects electric potentials associated with both intracellular as well as extracellular currents, radial and tangential to the surface of the recordings. Moreover, the spatial distribution of electric potentials is relatively smeared due to the passage through the tissues with different conductivities. This smearing is particularly important in the case of slow potential changes [9]. For those reasons, the physiological meaning of the positions and orientations of the CNV dipoles obtained in this study is more likely to be that of centers of the total distribution of electrical activity, rather than the exact cortical loci of the CNV generators. On the other hand, when MEG is used, a dipole representing the activity of an extended cortical surface is expected to lie in any sub-region of this surface, i.e., in its center or around the borders, depending on the particular geometry of the cortical currents in question, or even underneath the cortical surface, depending on the extension of the active area. Moreover, with MEG it is only a subset of cortical columns from the active ones that will contribute significantly to the scalp fields; it is only 
the portion of the cortex representing a breakdown in symmetry of current distribution in the total active surface that will result in a dipole-like field pattern on the scalp [6]. Finally, if the portion of cortex generating the dipole-like fields is large, the resulting equivalent current dipole will lie in a deeper position than the geometric center of the total active area [5]. Therefore, the equivalent current dipoles obtained with MEG as models of the magnetic equivalents of the CNVs are expected to be located closer to the active cortical areas (although not necessarily in their geometric center), when compared with those obtained with the EEG source localization method.

Various methods of investigation, including singlecell recordings [22] or surface-to-depth field potential analysis (e.g., [3]) in primates and metabolic tracing in humans [13], suggest that whenever changes in activity are observed in the prefrontal cortex, areas which are orders of magnitude larger than a single cortical column are the seat of activation. This fact, if valid, should lead to major differences in the results obtained by EEG and MEG when applied to slow electromagnetic changes in the brain. For instance, the simple rule stating that MEG detects the tangential component of the currents detected by EEG should not hold. The two methods are thus expected to index considerably different aspects of the same total electromagnetic changes occurring in an extended cortical patch. In particular, one should expect more spatial summation of electric as opposed to magnetic fields. This may explain the fact that a single dipole was obtained as the most stable model for each CNV in the present study. Thus, the single dipoles obtained for each task possibly represent the centers of the activity originating from the two hemispheres, from electrical configurations that are more widespread within each hemisphere, when compared with the part of those configurations corresponding to the primary current distributions that are detectable by MEG. Although the electric source analysis method that we used does not directly indicate the exact cortical areas that generate the CNVs, it can be used to demonstrate spatial differences in neural activity elicited by different tasks on each experimental subject. Therefore, this method can indirectly aid in the noninvasive functional mapping of the human frontal cortex.

\section{Acknowledgements}

The authors wish to thank Professor Andrew Papanicolaou and Dr. Dobrivoje S. Stokic for their critical review of the manuscript. This research was supported in part by grant NS 29540-005A1, from the National Institutes of Health, Bethesda, MD, and in part by the Vivian L. Smith Foundation for Restorative Neurology, Houston, TX, USA.

\section{References}

[1] L.F.H. Basile, R.L. Rogers, W.T. Bourbon and A.C. Papanicolaou, Slow magnetic fields from human frontal cortex, Electroencephalography and Clinical Neurophysiology 90 (1994), 157-165.

[2] P. Berg and M. Scherg, A fast method for forward computation of multiple-shell spherical head models, Electroencephalography and Clinical Neurophysiology 90 (1994), 5864.

[3] H. Gemba, K. Sasaki and T. Tsujimoto, Cortical field potential associated with hand movements triggered by warning and imperative stimuli in the monkey, Neuroscience Letters 113 (1990), 275-280.

[4] M.S. Hämäläinen and J. Sarvas, Realistic conductivity model of the human head for interpretation of neuromagnetic data, IEEE Transactions in Biomedical Engineering 36 (1989), $165-171$.

[5] L. Kaufman, B. Schwartz, C. Salustri and S.J. Williamson, Modulation of spontaneous brain activity during mental imagery, Journal of Cognitive Neuroscience 2 (1990), 124-132.

[6] L. Kaufman, J.H. Kaufman and J. Wang, On cortical folds and neuromagnetic fields, Electroencephalography and Clinical Neurophysiology 79 (1991), 211-226.

[7] J.D. Lewine, Neuromagnetic techniques for the noninvasive analysis of brain function, in: Noninvasive Techniques in Biology and Medicine, Chapter 3, S.E. Freeman, E. Fukushima and E.R. Greene, eds, San Francisco Press, San Francisco, 1990.

[8] W.C. McCallum, Potentials related to expectancy, preparation and motor activity, in: Handbook of Electroencephalography and Clinical Neurophysiology. Human Event-Related Potentials, T.W. Picton, ed., Elsevier, New York, 1988.

[9] P.L. Nunez, Electric Fields of the Brain: The Neurophysics of EEG, Oxford University Press, New York, 1981.

[10] R.L. Rogers, A.C. Papanicolaou, S.B. Baumann and H.M. Eisenberg, Late magnetic fields and positive evoked potentials following infrequent and unpredictable omissions of visual stimuli, Electroencephalography and Clinical Neurophysiology 83 (1992), 146-152.

[11] R.L. Rogers, L.F.H. Basile, A.C. Papanicolaou, T.W. Bourbon and H.M. Eisenberg, Visual evoked magnetic fields reveal activity in the superior temporal sulcus, Electroencephalography and Clinical Neurophysiology 86 (1993a), 344-347.

[12] R.L. Rogers, L.F.H. Basile, A.C. Papanicolaou and H.M. Eisenberg, Magnetoencephalography reveals two distinct sources associated with late positive evoked potentials during visual oddball tasks, Cerebral Cortex 3 (1993b), 163-169.

[13] P.E. Roland and L. Friberg, Localization of cortical areas activated by thinking, Journal of Neurophysiology 53 (1985), 1219-1243.

[14] M. Scherg and D. von Cramon, Two bilateral sources of the late AEP as identified by a spatio-temporal dipole model, Electroencephalography and Clinical Neurophysiology 62 (1985), 32-44. 
[15] M. Scherg and D. von Cramon, Evoked dipole source potentials of the human auditory cortex, Electroencephalography and Clinical Neurophysiology 65 (1986), 344-360.

[16] M. Scherg and P. Berg, Brain Electric Source Analysis Handbook.. Version 2.0.

[17] I.M. Tarkka, D.S. Stokic, L.F.H. Basile and A.C. Papanicolaou, Electric source localization of the auditory P300 agrees with magnetic source localization, Electroencephalography and Clinical Neurophysiology 96 (1995), 538-545.

[18] I.M. Tarkka, S. Micheloyannis, D.S. Stokic, Generators for human P300 elicited by somatosensory stimuli using multiple dipole source analysis, Neuroscience 75 (1996), 275-287.

[19] W.G. Walter and H.J. Crow, Depth recording from the human brain, Electroencephalography and Clinical Neurophysiology 16 (1964), 68-72.
[20] W.G. Walter, R. Cooper, V.J. Aldridge, W.C. McCallum and A.L. Winter, Contingent negative variation: an electric sign of sensorimotor association and expectancy in the human brain, Nature 203 (1964), 380-384.

[21] S.J. Williamson and L. Kaufman, Analysis of neuromagnetic signals, in: Handbook of Electroencephalography and Clinical Neurophysiology. Human Event-Related Potentials, A.S. Gevins and A. Remond, eds, Elsevier, New York, 1987.

[22] F.A.W. Wilson, S.P.O. Scalaidhe and P.S. Goldman-Rakic, Dissociations of object and spatial processing domains in primate prefrontal cortex, Science 260 (1993), 1955-1958. 


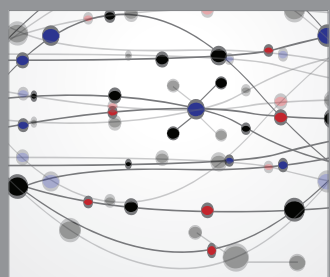

The Scientific World Journal
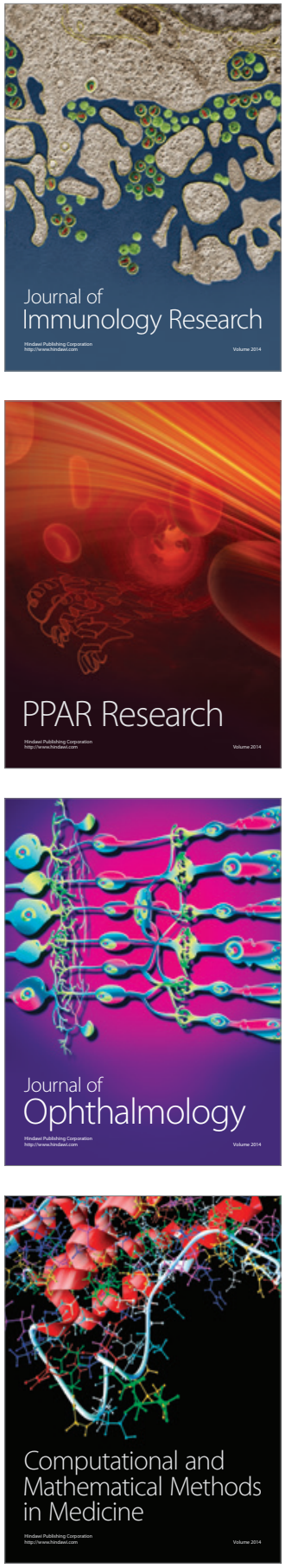

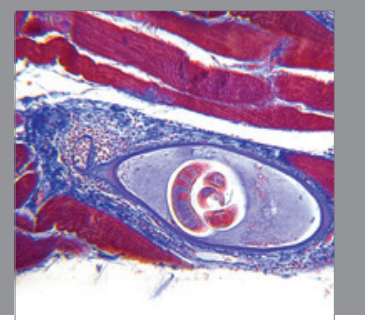

Gastroenterology

Research and Practice
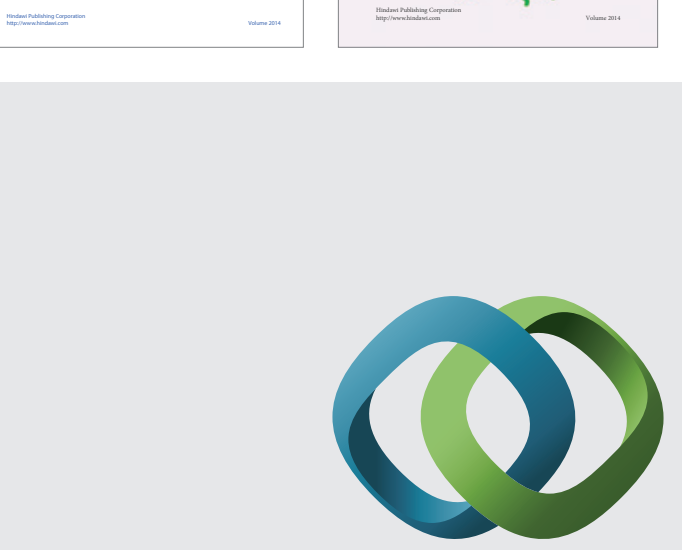

\section{Hindawi}

Submit your manuscripts at

http://www.hindawi.com
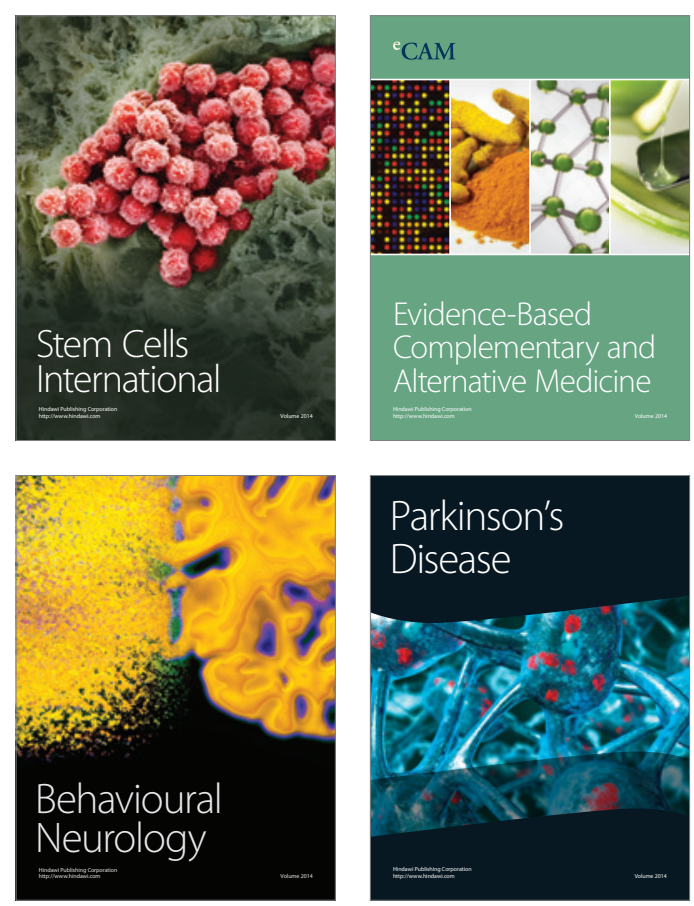

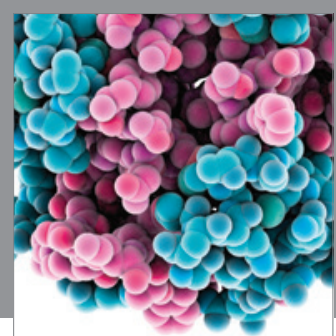

Journal of
Diabetes Research

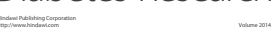

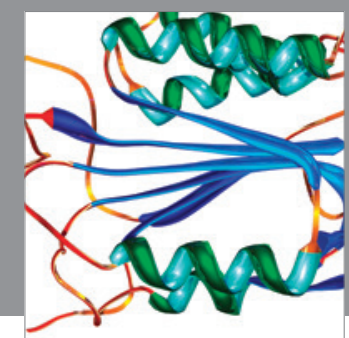

Disease Markers
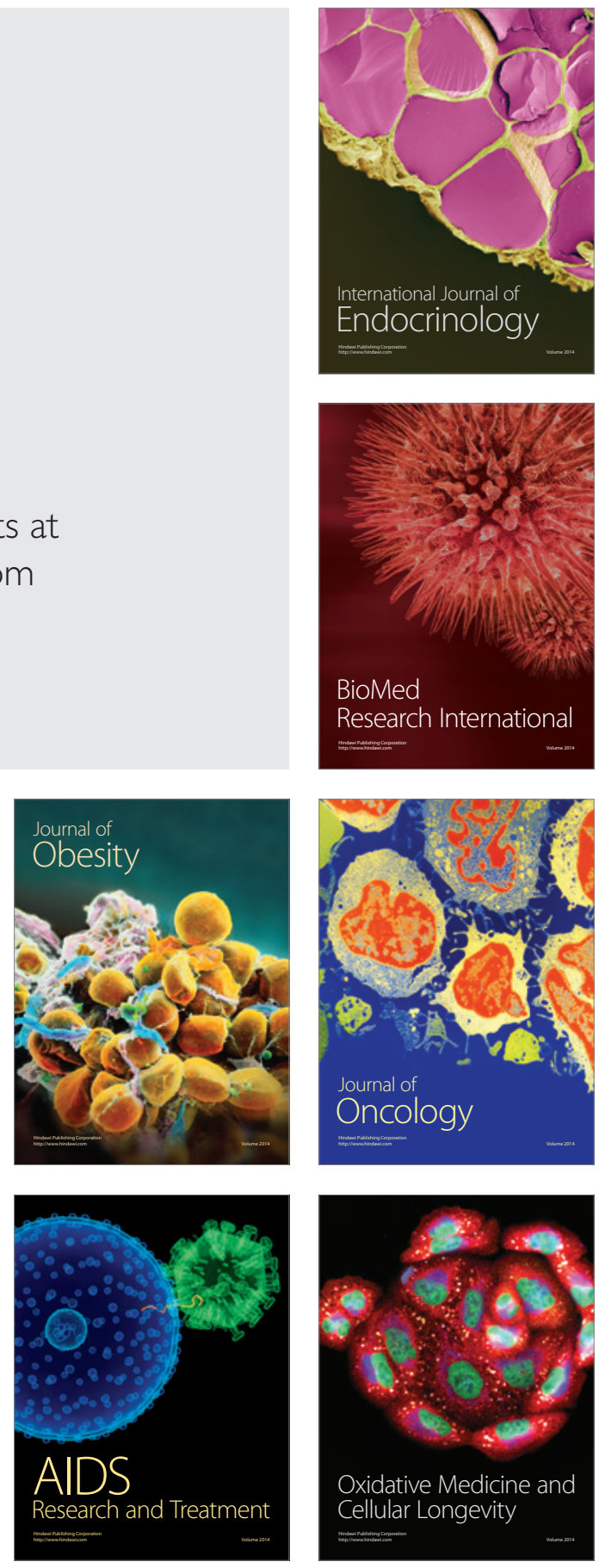